\title{
Symplectic Reflection Algebras
}

\author{
KENNETH A. BROWN
}

\section{INTRODUCTION}

1.1. In this paper I shall describe a beautiful class of associative algebras, the symplectic reflection algebras of the title, introduced in a recent paper of Etingof and Ginzburg [11]. These algebras are deformations of, and so retain some of the properties of, skew group algebras of finite groups, so it's with the latter class of algebras with which we begin in Section 2, in particular with the skew group algebra $S(V) * \Gamma$ of a finite group $\Gamma$ acting linearly on a finite dimensional complex vector space $V$, and hence acting on the symmetric algebra of $V, S(V)$.

One can deform such an algebra by destroying the commutativity of $S(V)$ using a skew-symmetric bilinear form $\kappa: V \times V \longrightarrow \mathbb{C} \Gamma$, as we explain in (4.1); but in general when this is done the algebra which results is too "small", in the sense that there is no natural vector space bijection with $S(V) * \Gamma$. Remarkably, however, if $\Gamma$ consists of symplectic automorphisms of the symplectic space $(V, \omega)$ then there is a class of forms $\kappa$ derived from $\omega$ for which the resulting deformations $d o$ have a natural linear bijection with $S(V) * \Gamma$ - that is, there is a "PBW theorem" in this setting, which we state and explain in (4.1). The definition of this class of forms requires the concept of a symplectic reflection, the analogue in the symplectic world of the pseudo-reflections on an ordinary vector space. So we define and discuss these symplectic reflections in Section 3, before going on to state the PBW theorem of Etingof and Ginzburg and

This is an expanded version of a talk given at All Ireland Algebra Days, Queen's University, Belfast, in May 2001. I am very grateful to the organising committee and in particular Dr M Mathieu for their generous hospitality and efficient organisation. All my own research on the topics described here has been joint with my colleague Dr Iain Gordon (University of Glasgow). 
give the resulting definition of symplectic reflection algebra in Section 4. From the definition we can quickly deduce in (4.4) that the symplectic reflection algebras are noetherian $\mathbb{C}$-algebras with many attractive algebraic and homological properties.

For a given $(V, \omega, \Gamma)$ with $\Gamma$ generated by symplectic reflections, the space of symplectic reflection algebras is parametrised by $(t, \mathbf{c}) \in$ $\mathbb{C} \times \mathbb{C}^{r} / \mathbb{C}^{*}$, where $r$ is the number of conjugacy classes of symplectic reflections in $\Gamma$. There is a dichotomy on this space, corresponding to the familiar special cases (when $V=\mathbb{C}^{2 m}$ ) of $S(V) * \Gamma$ and the skew group algebra of the Weyl algebra, $A_{m}(\mathbb{C}) * \Gamma$. Namely, every symplectic reflection algebra $H_{t, \mathbf{c}}$ is either a finite module over its centre, when $t=0$, or has centre the scalars $\mathbb{C}$ when $t$ is non-zero. I explain this dichotomy in (4.5), and go on in Section 5 to explain the key tool used in its proof. This is the idea of a Poisson bracket.

The centre $Z_{0, \mathbf{c}}$ of $H_{0, \mathbf{c}}$ admits a Poisson bracket; more generally this bracket extends to give an action of $Z_{0, \mathbf{c}}$ by derivations on $H_{0, \mathbf{c}}$, making $H_{0, \mathbf{c}}$ into a Poisson $Z_{0, \mathbf{c}}$-order. Further examples of Poisson orders are provided by most (if not all) quantum groups where the deformation parameter $q$ is a root of unity (5.4)4.

As well as being crucial for the proofs of many properties of $H_{t, \mathbf{c}}$, the presence of a Poisson structure on $Z_{0, \mathbf{c}}$, deforming the one given by the restriction of $\omega$ to $S(V)^{\Gamma}$, is one of the main reasons why symplectic reflection algebras are so interesting. For, on the one hand, this means that one can use these algebras to study the "symplectic deformations" of the quotient variety $V / \Gamma$; in particular one can seek a "symplectic desingularisation" of this space. On the other hand, the structure of Poisson order on $H_{0, \mathbf{c}}$ has drastic consequences for the representation theory of this algebra, thanks to the stratification of the variety maxspec $\left(Z_{0, \mathbf{c}}\right)$ by its symplectic leaves. We provide a sketch of some of these ideas in the remainder of Section 5 and in Section 6 .

\section{Skew Group Algebras}

2.1. Notation and Definitions. Throughout the paper $V$ will denote a finite dimensional complex vector space and $\Gamma$ will be a finite group acting linearly and faithfully as automorphisms of $V$, so there is a monomorphism of groups from $\Gamma$ into $G L(V)$. We'll write $v^{\gamma}$ for the action of $\gamma \in \Gamma$ on $v \in V$. Let $S(V)$ be the symmetric algebra on $V$. The skew group algebra

$$
H=S(V) * \Gamma
$$


is the associative $\mathbb{C}$-algebra which, as a vector space, is $S(V) \otimes_{\mathbb{C}} \mathbb{C} \Gamma$, with the usual multiplication on $S(V)$ and on $\mathbb{C} \Gamma$, and with

$$
\gamma v=v^{\gamma} \gamma
$$

for $v \in V$ and $\gamma \in \Gamma$.

Of course, such algebras are familiar and much studied objects, with books (for example [20]) devoted to them and their generalisations. It's an easy exercise to check that the centre of $H, Z(H)$, is given by the algebra $S(V)^{\Gamma}$ of $\Gamma$-invariants in $S(V)$. By a famous theorem [1, Theorem 1.3.1] of Hilbert (although the generalisation to arbitrary characteristic is due to Noether), $S(V)^{\Gamma}$ is itself an affine $\mathbb{C}$-algebra and $S(V)$ is a finitely generated $S(V)^{\Gamma}$-module. Thus $H$ is also a finitely generated $S(V)^{\Gamma}$-module. Being finitely generated over its centre, $H$ is a polynomial identity algebra, or PI-algebra for short, and the properties of $H$ are closely connected to those of $S(V)^{\Gamma}$. In fact, the study of $S(V)^{\Gamma}$ has always been one of the main reasons for studying $S(V) * \Gamma$; and one of the chief motives for introducing the deformations of $H$ which we'll come to below is to improve our understanding of the geometry of the variety $V / \Gamma$, whose coordinate ring is of course $S(V)^{\Gamma}$.

2.2. Example. Here is what is perhaps the simplest non-trivial case of the above construction. It is worth looking at now because it is one of the types we'll deform when we come to look at symplectic reflection algebras. Let $V$ have dimension 2, say

$$
V=\mathbb{C} x \oplus \mathbb{C} y .
$$

Let $n$ be an integer greater than one and let $\epsilon$ be a primitive $n$th root of unity. Set

$$
\gamma=\left(\begin{array}{rr}
\epsilon & 0 \\
0 & \epsilon^{-1}
\end{array}\right) \in S L_{2}(\mathbb{C})
$$

and

$$
\Gamma=\langle\gamma\rangle
$$

so that $\Gamma$ acts on $V$ with

$$
x^{\gamma}=\epsilon x \quad \text { and } \quad y^{\gamma}=\epsilon^{-1} y .
$$

Thus

$$
\begin{aligned}
H & =S(V) * \Gamma \\
& =\mathbb{C}\left\langle x, y, \gamma: x y=y x ; \gamma^{n}=1 ; \gamma x=\epsilon x \gamma ; \gamma y=\epsilon^{-1} y \gamma\right\rangle,
\end{aligned}
$$


and it is easy to check that

$$
S(V)^{\Gamma}=\mathbb{C}\left\langle x^{n}, y^{n}, x y\right\rangle
$$

Thus the ring of invariants is isomorphic to the factor of the polynomial algebra $\mathbb{C}[A, B, C]$ by the ideal generated by $A B-C^{n}$ - this is a Kleinian singularity of type A.

2.3. So far as the geometry of the quotient variety $V / \Gamma$ is concerned, the simplest possibility is that this variety is itself smooth (in contrast to Example (2.2)). In fact (since $S(V)^{\Gamma}$ is $\mathbb{N}$-graded with $S(V)_{0}^{\Gamma}=\mathbb{C}$ ) this can happen only when $S(V)^{\Gamma}$ is a polynomial algebra. Since it will help to motivate the later definition of symplectic reflections, let's recall the 1954 theorem of Shepherd and Todd which describes when this happens. An element $\gamma$ of $\Gamma$ is called a pseudo-reflection if the endomorphism $(1-\gamma)$ of $V$ has rank 1 ; equivalently, if 1 is an eigenvalue of $\gamma$ on $V$ of multiplicity $\operatorname{dim}(V)-1$. The groups which are generated by pseudo-reflections in their action on a complex vector space were classified by Shepherd and Todd [1, page 81 .

Theorem. ([1, Theorem 7.2.1]) $S(V)^{\Gamma}$ is a polynomial algebra if and only if $\Gamma$ is generated by pseudo-reflections for its action on $V$.

2.4. Skew Group Algebras over the Weyl Algebra. Recall that, for a positive integer $m$, the $m$ th Weyl algebra (over $\mathbb{C}), A_{m}(\mathbb{C})$, is the algebra of differential operators with polynomial coefficients on affine $m$-space $\mathbb{C}^{m}$, and so is the $\mathbb{C}$-algebra generated by the elements $\left\{x_{1}, \ldots, x_{m}, y_{1}, \ldots, y_{m}\right\}$, subject to the relations $y_{j} x_{i}-x_{i} y_{j}=\delta_{i j}$ and $x_{i} x_{j}-x_{j} x_{i}=0=y_{i} y_{j}-y_{j} y_{i} . A_{m}(\mathbb{C})$ is a simple noetherian $\mathbb{C}$-algebra, [12, Corollaries 1.13 and 1.15].

A variation of the construction from (2.1) which has also been well-studied in the past is obtained by replacing the symmetric algebra $S(V)$ by a Weyl algebra $A_{m}(\mathbb{C})$. So $\Gamma$ should in this case be a finite group of "linear" automorphisms of $A_{m}(\mathbb{C})$ : that is, $\Gamma$ should be a finite group of automorphisms of the vector space $V=\sum_{i} \mathbb{C} x_{i}+\sum_{j} \mathbb{C} y_{j}$ which preserves the non-singular alternating form $\omega$ on $V$ defined by $\omega\left(y_{j}, x_{i}\right)=\delta_{i j} ; \omega\left(x_{i}, x_{j}\right)=\omega\left(y_{i}, y_{j}\right)=0$. In other words, $\Gamma$ is a finite subgroup of the symplectic group $S p_{2 m}(\mathbb{C})$. In stark contrast to the "nearly commutative" situation of (2.1), $A_{m}(\mathbb{C}) * \Gamma$ is as far from being commutative as possible: $A_{m}(\mathbb{C}) * \Gamma$ is a simple ring [20, Theorem 15.8]. 
Notice that when $m=1, S p_{2}(\mathbb{C})=S L_{2}(\mathbb{C})$, so that the group $\Gamma$ of $(2.2)$ is in $S p_{2}(\mathbb{C})$. Thus there is a Weyl algebra version of Example (2.2), with the resulting algebra having the same generators and relations as (1), except that $y x-x y=1$.

\section{Symplectic Actions}

3.1. Symplectic Reflections. Symplectic reflections are the analogue in the symplectic world of pseudo-reflections in the Euclidean world. To define them, fix a finite dimensional symplectic $\mathbb{C}$-vector space $V$. Thus $V$ admits a non-degenerate alternating bilinear form $\omega$; $V$ has even dimension $2 m$, and we can choose a basis $\left\{x_{1}, \ldots, x_{m}\right.$, $\left.y_{1}, \ldots, y_{m}\right\}$ of $V$ such that $\omega$ is as in the previous paragraph. Now let $\Gamma$ be a finite subgroup of the symplectic group $S p(V)$ - that is, $\Gamma \subseteq G L(V)$ as before, with

$$
\omega\left(v^{\gamma}, u^{\gamma}\right)=\omega(v, u)
$$

for all $v, u \in V$ and $\gamma \in \Gamma$.

An element $s$ of $\Gamma$ is called a symplectic reflection (on $V$ ) if the endomorphism $1-s$ of $V$ has rank 2. We shall denote the set of symplectic reflections for the action of $\Gamma$ on $V$ by $S$. Clearly, $S^{\Gamma}=S$. We shall say that $\Gamma$ (in its action on $V$ ) is a symplectic reflection group if $\Gamma$ is generated by its symplectic reflections (on $V$ ). As the brackets suggest, we'll suppress mention of $V$ where the vector space and action are clear from the context.

3.2. Examples. 1. Kleinian singularities. Let $m=1$, so that $V=\mathbb{C} x \oplus \mathbb{C} y$ with $\omega(y, x)=1$. As observed in (2.4), $S p(V)=$ $S L(V)$. Moreover, if $\Gamma$ is a finite subgroup of $S L(V)$ and $1 \neq s \in \Gamma$, then $s$ can't have 1 as an eigenvalue $(\operatorname{since} \operatorname{det}(s)=1)$, so that $s$ is a symplectic reflection. Therefore $\Gamma$ is a symplectic reflection group.

2. Dual pairs. Let $U$ be a finite dimensional $\mathbb{C}$-vector space, and let $W$ be a finite subgroup of $G L(U)$. Suppose that $W$ is generated by pseudo-reflections in its action on $U$, in the sense of (2.3). So the contragredient action of $W$ on $U^{*}$ is also generated by pseudoreflections. Then $V:=U \oplus U^{*}$ is symplectic, with

$$
\omega((y, f),(u, g))=g(y)-f(u)
$$

for $y, u \in U$ and $f, g \in U^{*}$. And it's clear that the pseudo-reflections for the action of $W$ on $U$ are symplectic reflections for the action on $V$, so that $W$ is a symplectic reflection group on $V$. 
3.3. Classification. In [22, Question 1.3], M. Verbitsky posed the Problem: Classify the (complex) symplectic reflection groups.

There is a natural notion of an indecomposable symplectic triple $(V, \omega, \Gamma)$ : the triple is indecomposable if there is no non-trivial decomposition $V=U \oplus W$ of $V$ into $\Gamma$-stable symplectic subspaces. It's routine to reduce the above problem to consideration of indecomposable triples. Following the statement of this problem in my talk at All Ireland Algebra Days, R. Guralnick was able to carry out the classification using results from his joint paper with J. Saxl [13]; details will appear as an Appendix to [13]. The result (with details omitted) is as follows.

Theorem. (Guralnick-Saxl) Let $(V, \omega, \Gamma)$ be an indecomposable symplectic triple.

1. If $V$ is not an irreducible $\mathbb{C} \Gamma$-module, then $(V, \omega, \Gamma)$ is a dual pair as in (3.2)2, with $U$ an irreducible $\mathbb{C} \Gamma$-module.

2. If $V$ is an irreducible $\mathbb{C} \Gamma$-module, then one of the following holds:

(i) $V=V_{1} \perp \ldots \perp V_{r}$ where each $V_{i}$ is a 2-dimensional symplectic subspace of $V$ and $\Gamma$ permutes the $V_{i}$ as $S_{r}$. Moreover, $H$, the image of the stabilizer of $V_{1}$ in $G L\left(V_{1}\right)$, is an irreducible subgroup of $S p\left(V_{1}\right)$ and $\Gamma$ embeds in $H$ ? $S_{r}$;

or

(ii) $(V, \omega, \Gamma)$ is one of a finite list of explicit examples, with $\operatorname{dim}_{\mathbb{C}}(V)$ at most 10 .

3.4. Symplectic Geometry. Let $(V, \omega)$ be a finite dimensional symplectic $\mathbb{C}$-vector space and let $\Gamma$ be a finite subgroup of $S p(V)$. Then the smooth locus of the quotient variety $X:=V / \Gamma$ admits a natural symplectic form $\tilde{\omega}$ induced from $\omega$. A symplectic resolution of $X$,

$$
\pi: \tilde{X} \longrightarrow X,
$$

is a resolution of singularities of $X$ such that the pull-back $\pi^{*} \tilde{\omega}$ of $\tilde{\omega}$ extends to a holomorphic symplectic form on $\tilde{X}$. There is a great deal of current interest in symplectic desingularisation, motivated by developments in differential and algebraic geometry and in theoretical physics. One fundamental problem which remains open is:

Problem: For which symplectic quotient singularities as defined above does a symplectic resolution exist?

Here is what is known at present about this question: 
Theorem. Let $V, \omega$ and $\Gamma$ be as above, and set $X=V / \Gamma$.

1. (Du Val, [10]) If $V$ has dimension 2 (that is, the Kleinian case), then $X$ has a symplectic resolution.

2. (Verbitsky, [22, Theorem 3.2]) If $X$ has a symplectic resolution then $\Gamma$ is a symplectic reflection group.

3. (Kaledin, [15, Sec.6]) The converse to 2 is false. A counterexample is provided by the dual pair (see Example (3.2).2) of type $G_{2}$, so that $\Gamma$ is $\mathbb{Z} / 2 \mathbb{Z} \times S_{3}$ and $V$ has dimension 4 .

We shall gain tantalising hints in later sections that the above problem and the partial solutions outlined in the theorem are closely linked to the structure of the noncommutative algebras which we are about to define - the symplectic reflection algebras of the title.

\section{Symplectic Reflection Algebras}

4.1. Definition and PBW Theorem. Let $V$ and $\Gamma$ be as in (2.1), and let

$$
\kappa: V \times V \longrightarrow \mathbb{C} \Gamma
$$

be a skew-symmetric bilinear form. Let

$$
T(V):=\mathbb{C} \oplus V \oplus(V \otimes V) \oplus \ldots \oplus V^{\otimes n} \oplus \ldots
$$

be the tensor algebra of $V$. Just as with $S(V)$, the action of $\Gamma$ on $V$ extends to an action of $\Gamma$ on $T(V)$ by $\mathbb{C}$-algebra automorphisms, and so we can construct the skew group algebra $T(V) * \Gamma$ in the same way as we formed $S(V) * \Gamma$ in (2.1). Now define a factor algebra $H_{\kappa}$ of $T(V) * \Gamma$ by

$$
H_{\kappa}:=T(V) * \Gamma /\langle x y-y x-\kappa(x, y): x, y, \in V\rangle .
$$

Examples: 1. If $\kappa \equiv 0$ then we recover the skew group algebra $S(V) * \Gamma$ of $(2.1)$, now denoted by $H_{0}$.

2. Suppose that $V$ is the standard symplectic space $(V, \omega)$ of dimension $2 m$ described in (3.1), and take $\kappa=\omega$ (where of course we regard $\mathbb{C}$ as embedded in $\mathbb{C} \Gamma$ via the map $\left.c \mapsto c 1_{\Gamma}\right)$. Then $H_{\kappa}$ is the skew group algebra $A_{m}(\mathbb{C}) * \Gamma$ of $(2.4)$.

For a general $\kappa, H_{\kappa}$ is a filtered $\mathbb{C}$-algebra - that is, there are finite dimensional vector subspaces $F_{i}$ of $H_{\kappa}$, for $i \geq 0$, with $F_{i} \subseteq F_{i+1}$ and $F_{i} F_{j} \subseteq F_{i+j}$ for all $i$ and $j$, and $H_{\kappa}=\cup_{i \geq 0} F_{i}$. Namely, set

$$
F_{0}=\mathbb{C} \Gamma ; \quad F_{1}=\mathbb{C} \Gamma+\mathbb{C} \Gamma V ; \quad \text { and } \quad F_{i}=\left(F_{1}\right)^{i}, \quad \text { for } \quad i \geq 1 .
$$


We can thus form the associated graded ring $\operatorname{gr}\left(H_{\kappa}\right)$ of $H_{\kappa}$,

$$
\operatorname{gr}\left(H_{\kappa}\right):=\bigoplus_{i \geq 0}\left(F_{i+1} / F_{i}\right)
$$

Clearly there is an epimorphism of algebras

$$
\rho: S(V) * \Gamma \rightarrow \operatorname{gr}\left(H_{\kappa}\right) .
$$

We shall say that $P B W$ holds for $H_{\kappa}$ if $\rho$ is an isomorphism; equivalently, if $H_{\kappa}=H_{0}=S(V) * \Gamma$ as $\mathbb{C}$-vector spaces. In particular, PBW holds for both of the examples $S(V) * \Gamma$ and $A_{m}(\mathbb{C}) * \Gamma$ given above. There now arises the natural question: for which $(V, \Gamma, \kappa)$ does PBW hold? The answer to this question in the case of symplectic spaces and actions is surprisingly elegant and includes a huge range of very interesting algebras, as we shall now see. From now on, we assume that $(V, \omega, \Gamma)$ are as in (3.1). Note that for $\gamma \in \Gamma$ there is a $\omega$-orthogonal decomposition $V=\operatorname{Im}(1-\gamma) \oplus \operatorname{Ker}(1-\gamma)$. For $s$ in the set $S$ of symplectic reflections in $\Gamma$, write $\omega_{s}$ for the skew-symmetric form on $V$ which has $\operatorname{Ker}(1-s)$ as its radical, and coincides with $\omega$ on $\operatorname{Im}(1-s)$.

Theorem. (Etingof-Ginzburg, [11, Theorem 1.3]) Let $(V, \omega, \Gamma)$ be an indecomposable symplectic triple, and let $\kappa: V \times V \longrightarrow \mathbb{C} \Gamma$ be a skew-symmetric bilinear form. Then $P B W$ holds for $H_{\kappa}$ if and only if there exist a constant $t \in \mathbb{C}$ and a function $c: S \longrightarrow \mathbb{C}: s \mapsto c_{s}$, with $c$ constant on $\Gamma$-conjugacy classes, such that

$$
\kappa(x, y)=t \omega(x, y) 1_{\Gamma}+\sum_{s \in S} c_{s} \omega_{s}(x, y) s
$$

for all $x, y \in V$.

In the light of the theorem one is led to make the

Definition: Let $(V, \omega, \Gamma)$ be an indecomposable symplectic triple, and let $\kappa$ be a form which conforms to the description in the theorem. Then $H_{\kappa}$ is called a symplectic reflection algebra.

4.2. First Properties. Observe that the subgroup $T:=\langle S\rangle$ is normal in $\Gamma$, so that, for $\kappa$ as in the definition just given,

$$
H_{\kappa}=H_{\kappa}(T) *(\Gamma / T),
$$

where $H_{\kappa}(T)$ denotes the symplectic reflection algebra constructed using $T$ in place of $\Gamma$ (but with all the other ingredients unchanged), 
and the right hand side denotes a crossed product of $\Gamma / T$ with coefficients from $H_{\kappa}(T)$. (See [20] for the definition of crossed product.) Hence it makes sense to assume that $\Gamma$ is generated by its symplectic reflections when studying a symplectic reflection algebra $H_{\kappa}$.

Let the $\Gamma$-conjugacy classes of symplectic reflections be $\mathcal{C}_{1}, \ldots, \mathcal{C}_{r}$, let $t \in \mathbb{C}$ and let $\mathbf{c}=\left(c_{1}, \ldots, c_{r}\right) \in \mathbb{C}^{r}$. Then we'll write $H_{t, \mathbf{c}}$ instead of $H_{\kappa}$ when $\kappa$ is given as in (3), with $c(s)=c_{i}$ for $s \in \mathcal{C}_{i}$. In particular, we recover the special cases considered in (2.1) and (2.4) as

$$
H_{0, \mathbf{0}} \cong S(V) * \Gamma \quad \text { and } \quad H_{1, \mathbf{0}} \cong A_{m}(\mathbb{C}) * \Gamma .
$$

It's very easy to see from (2) and (3) that $H_{t, \mathbf{c}}$ is isomorphic to $H_{\lambda t, \lambda \mathbf{c}}$ for all $\lambda \in \mathbb{C}^{*}$. Thus what we have obtained by means of the construction just defined is a collection of deformations of $S(V) * \Gamma$ parametrised by $\mathbb{C}^{r+1} / \mathbb{C}^{*}=\mathbb{P}^{r}(\mathbb{C})$.

4.3. Example. Let $V$ and $\Gamma$ be as in (2.2). As we observed in (3.2), this example is symplectic if we set $\omega(x, y)=1$, and every non-identity element of $\Gamma$ is a symplectic reflection. Thus, for each choice of $t \in \mathbb{C}$ and $\mathbf{c}=\left(c_{1}, \ldots, c_{n-1}\right) \in \mathbb{C}^{n-1}$, we get a symplectic reflection algebra

$$
\begin{gathered}
H_{t, \mathbf{c}}=\mathbb{C}\left\langle x, y, \gamma: \gamma^{n}=1, \gamma x=\epsilon x \gamma, \gamma y=\epsilon^{-1} y \gamma,\right. \\
\left.y x-x y=t+\sum_{i=1}^{n-1} c_{i} \gamma^{i}\right\rangle .
\end{gathered}
$$

4.4. Consequences of the Filtration. Being a finite module over $S(V), S(V) * \Gamma$ is a noetherian algebra, and faithfulness of the $\Gamma$ action on $V$ ensures that $S(V) * \Gamma$ is prime [20, Corollary 12.6]. Since $H_{t, \mathrm{c}}$ is $\mathbb{N}$-filtered with $S(V) * \Gamma$ as associated graded ring, as follows from the discussion and definition in (4.1), standard filtered-graded techniques $[19,1.6 .3,1.6 .9]$ can be used to see that these prime and noetherian properties pass to $H_{t, \mathbf{c}}$. Similarly, thanks to Hilbert's syzygy theorem [19, Theorem 7.5.3] and the generalised Maschke theorem [20, Theorem 4.1], $S(V) * \Gamma$ has finite global (homological) dimension; and this transfers also to $H_{t, \mathbf{c}}$ by [19, Corollary 7.6.18]. In fact, the more sophisticated homological properties of being Auslander-regular and Cohen-Macaulay (which we won't define here, see for example [17]) can also be deduced for $H_{t, \mathbf{c}}$ as a consequence of the fact that they hold for $S(V) * \Gamma$. (Imitate the proof for the case $\operatorname{dim}(V)=2$ given in [5, Theorem 1.5].) Finally, when $\Gamma$ is a symplectic reflection group, $S(V) * \Gamma$ is a maximal order by 
$[18$, Theorem 4.6], and a prime noetherian $\mathbb{N}$-filtered ring whose associated graded ring is a maximal order is itself a maximal order, by [21], so that $H_{t, \mathbf{c}}$ is a maximal order in its simple artinian quotient ring. (For the definition of a maximal order, see [19, 5.1].

We summarise the above in the

Theorem. Let $(V, \omega, \Gamma)$ be an indecomposable symplectic triple, with $\Gamma$ generated by its symplectic reflections as in (4.2). Let $t \in \mathbb{C}$ and $\mathbf{c} \in \mathbb{C}^{r}$. Then $H_{t, \mathbf{c}}$ is a prime noetherian maximal order which is Auslander-regular and Cohen-Macaulay.

4.5. The Symmetrising Idempotent and the Centre of $H_{t, \mathbf{c}}$. The symmetrising idempotent is the element

$$
e=\frac{1}{|\Gamma|} \sum_{\gamma \in \Gamma} \gamma \in \mathbb{C} \Gamma \text {. }
$$

Since $\mathbb{C} \Gamma \subseteq H_{t, \mathbf{c}}$ for all choices of $t$ and $\mathbf{c}, e$ is an idempotent element of every $H_{t, \mathbf{c}}$, (and we'll use the same symbol $e$ for this element in every case). Denote the centre of the algebra $A$ by $Z(A)$. It's wellknown (and an easy exercise) that

$$
e S(V) * \Gamma e \cong S(V)^{\Gamma}=Z\left(H_{0,0}\right) .
$$

Fix $t \in \mathbb{C}$ and $\mathbf{c} \in \mathbb{C}^{r}$. The filtration $\left\{F_{i}\right\}$ of (4.1) intersects with $e H_{t, \mathbf{c}} e$ to give an $\mathbb{N}$-filtration of the latter ring (since $e \in \mathbb{C} \Gamma=F_{0}$ ), and

$$
\operatorname{gr}\left(e H_{t, \mathbf{c}} e\right)=\operatorname{egr}\left(H_{t, \mathbf{c}}\right) e=e S(V) * \Gamma e \cong S(V)^{\Gamma} .
$$

It's worth emphasising this point by stating it another way, since it's one of the fundamental reasons for the importance of the algebras $H_{t, \mathbf{c}}: e H_{t, \mathbf{c}} e$ is a deformation of the quotient singularity $S(V)^{\Gamma}$, for every choice of $t$ and $\mathbf{c}$.

There is a second vital feature of $e H_{t, \mathbf{c}} e$ : its structure determines the centre of $H_{t, \mathbf{c}}$. This is the first part of the following result.

Theorem. Fix an indecomposable symplectic triple $(V, \omega, \Gamma)$ with $\Gamma$ generated by symplectic reflections, and let $t \in \mathbb{C}$ and $\mathbf{c} \in \mathbb{C}^{r}$.

1. The map $H_{t, \mathbf{c}} \longrightarrow e H_{t, \mathbf{c}} e: h \mapsto$ ehe restricts to an algebra isomorphism $\theta$ of $Z_{t, \mathbf{c}}:=Z\left(H_{t, \mathbf{c}}\right)$ with $Z\left(e H_{t, \mathbf{c}} e\right)$.

2. $H_{t, \mathbf{c}}$ satisfies a polynomial identity if and only if $H_{t, \mathbf{c}}$ is a finite module over its centre if and only if $t=0$.

3. Suppose that $t=0$. Then $\operatorname{im}(\theta)=e H_{t, \mathbf{c}} e$, so that

$$
Z_{0, \mathbf{c}} \cong e H_{0, \mathbf{c}} e \text {. }
$$


That is, (in view of (5)), $Z_{0, \mathbf{c}}$ is a commutative deformation of $S(V)^{\Gamma}$.

4. When $t \neq 0, Z_{t, \mathbf{c}}=\mathbb{C}$.

Parts 1 and 3 and the sufficiency of $t=0$ in 2 are due to Etingof and Ginzburg [11, Theorems 3.1 and 1.6]. The remainder is due to Brown and Gordon [4]. In the case where $\operatorname{dim}(V)=2$ all the above results are due to Crawley-Boevey and Holland [5]. For the proof of 1 , it's trivial that $\theta$ is an algebra homomorphism. One proves that $\theta$ is an isomorphism by constructing an inverse map, the key point here being that $\operatorname{End}_{e H_{0, \mathbf{c}} e}\left(H_{0, \mathbf{c}} e\right) \cong H_{0, \mathbf{c}}$, which follows from the fact that $H_{0, \mathbf{c}}$ is a maximal order, Theorem (4.4). The other parts of the theorem are proved using the Poisson structures which we'll discuss in Section 5.

4.6. Example continued. Let's revisit Example (4.3) in the light of the above results. Let the primitive idempotents of $\mathbb{C} \Gamma$ be $e_{0}, \ldots$, $e_{n-1}$, with $e_{0}$ being the symmetrising idempotent $\frac{1}{n} \sum_{i=0}^{n} \gamma^{i}$. It's convenient to rewrite the relation defining $H_{t, \mathbf{c}}$ in (4) as

$$
y x-x y=\sum_{i=0}^{n-1} f_{i} e_{i},
$$

where $f_{i} \in \mathbb{C}$ for $i=0, \ldots, n-1$. The condition $t=0$ of Theorem (4.5) 2 is then restated as

$$
\sum_{i=0}^{n-1} f_{i}=0
$$

- think in terms of traces on the regular $\mathbb{C} \Gamma$-module. And one calculates easily that, when (6) holds,

$$
\begin{aligned}
e_{0} H_{0, \mathbf{c}} e_{0} & =\mathbb{C}\left\langle x^{n} e_{0}, y^{n} e_{0}, x y e_{0}\right\rangle \\
& \cong \mathbb{C}\left[A, B, H: A B=H \prod_{i=1}^{n-1}\left(H+f_{1}+\ldots+f_{i}\right)\right] .
\end{aligned}
$$

Notice that the centre is thus filtered, with

$$
\operatorname{deg}\left(x^{n} e_{0}\right)=\operatorname{deg}\left(y^{n} e_{0}\right)=n, \quad \operatorname{deg}\left(x y e_{0}\right)=2,
$$

so its associated graded ring is isomorphic to $\mathbb{C}\left[A, B, H: A B=H^{n}\right]$, which is $S(V)^{\Gamma}$ in this case, as predicted in (5). Notice also that the 
centre is smooth here if and only if the polynomial equation

$$
H \prod_{i=1}^{n-1}\left(H+f_{1}+\ldots+f_{i}\right)=0
$$

has no repeated roots.

\section{Poisson Structures}

The rich structure of the algebras $H_{t, \mathbf{c}}$ and $e H_{t, \mathbf{c}} e$ stems in large part from the fact that associated graded algebras of these algebras (and indeed the algebras themselves when $t=0$ ) admit Poisson structures. For example, the proof of Theorem (4.5) makes crucial use of these structures. We provide a sketch of the key ideas in this section.

5.1. Poisson Orders. Let $H$ be a finitely generated $\mathbb{C}$-algebra, finitely generated as a module over a central subalgebra $Z_{0}$. By the Artin-Tate Lemma $[19,13.9 .10], Z_{0}$ is itself a finitely generated $\mathbb{C}$-algebra. Denote the Lie algebra of $\mathbb{C}$-derivations of $H$ by $\operatorname{Der}_{\mathbb{C}}(H)$. Let's suppose that there is a linear map

$$
D: Z_{0} \longrightarrow \operatorname{Der}_{\mathbb{C}}(H): z \mapsto D_{z},
$$

satisfying

(a) $D_{z z^{\prime}}=z D_{z^{\prime}}+z^{\prime} D_{z}$ for all $z, z^{\prime} \in Z_{0}$;

(b) $Z_{0}$ is stable under $D\left(Z_{0}\right)$;

(c) the resulting bracket $\{\mid\}: Z_{0} \times Z_{0} \longrightarrow Z_{0}$, defined by $\left\{z \mid z^{\prime}\right\}=D_{z}\left(z^{\prime}\right)$, is a Lie bracket.

Then we shall say that $H$ is a Poisson $Z_{0}$-order. In this case $Z_{0}$ is a Poisson algebra, or, in other words, $\mathcal{Z}=\operatorname{maxspec}\left(Z_{0}\right)$ is a Poisson variety.

5.2. Quantization. Here is one important mechanism giving rise to a Poisson order. Let $\hat{H}$ be a $\mathbb{C}$-algebra, $\hat{Z}$ a subalgebra, and $t$ a central non-zero divisor of $\hat{H}$, contained in $\hat{Z}$. Assume that $Z_{0}=\hat{Z} / t \hat{Z}$ is a finitely generated central subalgebra of $H=\hat{H} / t \hat{H}$, and that $H$ is a finitely generated $Z_{0}$-module. Let $\pi: \hat{H} \longrightarrow H$ be the quotient map.

Given any $z \in Z_{0}$ we have a derivation of $H$, denoted $D_{z}$, defined by

$$
D_{z}(h)=\pi([\hat{z}, \hat{h}] / t),
$$


where $\hat{z} \in \hat{Z}$ and $\hat{h} \in \hat{H}$ are preimages under $\pi$ of $h$ and $z$ respectively. This makes sense because $[\hat{z}, \hat{h}] \in t \hat{H}$. The mapping $D: Z_{0} \longrightarrow \operatorname{Der}(H)$, given by $z \mapsto D_{z}$, satisfies the hypotheses of (5.1), [14].

5.3. Filtered and Graded Algebras. An important variant of the above is the following. Let $H$ be an $\mathbb{N}$-filtered $\mathbb{C}$-algebra whose $i^{\text {th }}$-filtered piece is denoted $F^{i} H$. Let $Z$ be a subalgebra of $H$, and give it the induced filtration. Denote the associated graded rings of $Z$ and $H$ by $\operatorname{gr} Z$ and $\operatorname{gr} H$ respectively. Suppose that $\operatorname{gr} Z$ is a finitely generated central subalgebra of $\operatorname{gr} H$, such that $\operatorname{gr} H$ is a finitely generated $\operatorname{gr} Z$-module. Let $\sigma_{i}: F^{i} H \longrightarrow \operatorname{gr} H$ be the $i^{\text {th }}$ principal symbol map, sending an element of $F^{i} H \backslash F^{i-1} H$ to its leading term. Given a graded element of $\operatorname{gr} Z$, say $\sigma_{m}(z)$, there is a well-defined derivation of $\operatorname{gr} H$, denoted $D_{\sigma_{m}(z)}$, given by

$$
D_{\sigma_{m}(z)}\left(\sigma_{n}(h)\right)=\sigma_{m+n-1}([z, h]) .
$$

Extending this linearly yields a mapping $D: \operatorname{gr} Z \longrightarrow \operatorname{Der}(\operatorname{gr} H)$, satisfying the hypotheses of (5.1).

To see that this is really a special case of (5.2), form the Rees algebras $\hat{H}=\bigoplus_{i} F^{i} H t^{i} \subseteq H[t]$ and $\hat{Z}=\bigoplus_{i} F^{i} Z t^{i} \subseteq Z[t]$, where $t$ is a central indeterminate. It can easily be checked that we recover the derivations in (7) from the construction in (5.2).

5.4. Examples. 1. Let $\left(V=\sum_{i=1}^{m} \mathbb{C} x_{i}+\mathbb{C} y_{i}, \omega, \Gamma\right)$ be an indecomposable symplectic triple as in (3.3), with the form defined as in (2.4). Then of course $S(V)$ is a Poisson algebra under the extension of the form on $V$ (using the Leibniz rule (5.1)(a)) to the "standard" Poisson bracket $\{-,-\}$ on $S(V)$. The $\Gamma$-invariance of $\omega$ forces $\Gamma$-invariance of $\{-,-\}$, and this implies that $S(V)^{\Gamma}$ is a Poisson subalgebra of $S(V)$. This definition extends to give a structure of Poisson $S(V)^{\Gamma}$-order on $H=S(V) * \Gamma$ if we set $D_{z}(\gamma)=0$ for $z \in Z(H)=S(V)^{\Gamma}$ and $\gamma \in \Gamma$.

Notice that this construction is a case of quantization (5.2). For, starting with $(V, \omega, \Gamma)$ as above and letting $t$ be an indeterminate, we can take $\hat{H}$ to be the $\mathbb{C}[t]$-algebra with the same relations as $H=S(V) * \Gamma$, except that we now require

$$
\left[x_{i}, y_{j}\right]=\delta_{i j} t
$$

for $i, j=1, \ldots, m$, instead of $\left[x_{i}, y_{j}\right]=0$. And in a similar way the construction is also an example of the filtered-graded case (5.3). 
2. $H_{0, \mathbf{c}}$ is a Poisson $Z_{0, \mathbf{c}}$-order. $\operatorname{Keep}(V, \omega, \Gamma)$ as in (5.4)1, but assume now that $\Gamma$ is generated by symplectic reflections in its action on $V$, let $r$ be the number of conjugacy classes of symplectic reflections in $\Gamma$, and fix $\mathbf{c} \in \mathbb{C}^{r}$. Now let $\hat{H}_{\mathbf{c}}$ be the $\mathbb{C}[t]$-algebra defined exactly as in (4.1), with relations as in (2) and (3), but with $t$ in (3) being now an indeterminate rather than a scalar. So now we are in the setting of (5.2), with $\hat{H}=\hat{H}_{\mathbf{c}}$, and $\hat{H} / t \hat{H} \cong H_{0, \mathbf{c}}$. Also, $Z_{0}=Z_{0, \mathbf{c}}$, and we take the subalgebra $\hat{Z}$ of $\hat{H}$ to be the inverse image of $Z_{0}$ under the canonical epimorphism from $\hat{H}$ onto $H_{0, \mathbf{c}}$. By Theorem (4.5)3, the conditions of (5.2) are fulfilled, and so $H_{0, \mathbf{c}}$ is a Poisson $Z_{0, \mathbf{c}^{-}}$order.

3. Quantum groups. Both the quantised enveloping algebras $\mathcal{U}_{\epsilon}(\mathfrak{g})$ and the quantised function algebras $\mathcal{O}_{\epsilon}(G)$ are Poisson orders when $\epsilon$ is a root of unity, thanks to the mechanism of 5.2. In fact the central Poisson subalgebra can be taken to be a Hopf subalgebra in these instances. For details and references, see [2], [6], [7].

5.5. Filtered and Graded Poisson Orders. Suppose that $Z_{0} \subseteq$ $H$ is a Poisson $Z_{0}$-order with non-zero bracket

$$
\{-,-\}: Z_{0} \times H \longrightarrow H \text {. }
$$

Suppose that $H$ is $\mathbb{N}$-filtered with $i$ th filtered subspace $F^{i} H$, and set $F^{i} Z_{0}=F^{i} H \cap Z_{0}$ for all $i \geq 0$. We say that $\{-,-\}$ has degree $d$ if

$$
\left\{F^{i} Z_{0}, F^{j} H\right\} \subseteq F^{i+j+d} H
$$

for all $i$ and $j$, and there exist $i, j \in \mathbb{N}$ and $z \in F^{i} Z_{0}, z^{\prime} \in F^{j} Z_{0}$ such that $\left\{z, z^{\prime}\right\}$ does not belong to $F^{i+j+d-1} H$. Then we can define a structure of Poisson gr $Z_{0}$-order on gr $H$ by setting, for $z \in F^{m} Z_{0}$ and $h \in F^{l} H$,

$$
\left\{\sigma_{m}(z), \sigma_{l}(h)\right\} \quad:=\sigma_{m+l+d}(\{z, h\}),
$$

where $\sigma_{i}$ for $i \geq 0$ denotes the appropriate principal term map. Conditions (5.1)(a),(b) and (c) are easy to check. Observe that the Poisson structure induced in this way on $\operatorname{gr} Z_{0}$ has degree precisely $d$.

5.6. Poisson Deformations. It's not hard to see that the Poisson structure on $H_{0, \mathbf{c}}$ defined in (5.4)2 has degree -2 . So (5.5) applies to the Poisson $Z_{0, \mathbf{c}}$-order $H_{0, \mathbf{c}}$, yielding a structure of Poisson $S(V)^{\Gamma_{-}}$ order of degree -2 on $H_{0,0}=S(V) * \Gamma$, and in particular a degree -2 Poisson structure on $S(V)^{\Gamma}$. However, it's a consequence of Hartog's theorem (see [11, Lemma 2.23(i)]) that (up to a scalar multiple) the 
only degree -2 Poisson bracket on $S(V)^{\Gamma}$ is the restriction (5.4)1 of the bracket on $S(V)$ induced by the symplectic form on $V$. We therefore obtain a more precise version of Theorem (4.5)3:

Theorem. The Poisson $Z_{0, \mathbf{c}}$-order structure of $H_{0, \mathbf{c}}$ of (5.4)2 is a deformation of the "standard" Poisson $Z_{0,0}$-structure on $H_{0,0}$ of (5.4)1.

5.7. Poisson Ideals and Subsets. A two-sided ideal $I$ of the Poisson $Z_{0}$-order $H$ (respectively $J$ of $Z_{0}$ ) is called Poisson if it is stable under $D\left(Z_{0}\right)$. Thanks to $[9,3.3 .2]$ if $I$ (respectively $J$ ) is Poisson then so too are both $\sqrt{I}$ (respectively $\sqrt{J}$ ) and the minimal prime ideals of $H$ (respectively $Z_{0}$ ) over $I$ (respectively $J$ ). We shall denote the space of prime Poisson ideals of $Z_{0}$, with the topology induced from the Zariski topology on $\operatorname{spec}\left(Z_{0}\right)$, by $\mathcal{P}-\operatorname{spec}\left(Z_{0}\right)$. Clearly, if $I$ is a Poisson ideal of $Z_{0}$ then there is an induced structure of Poisson algebra on $Z_{0} / I$. We shall denote the maximal ideal spectrum of $Z_{0}$ by $\mathcal{Z}$. For a semiprime ideal $I$ of $Z_{0}$ we write $\mathcal{V}(I)$ for the closed subset of $\mathcal{Z}$ defined by $I$. A closed subset $\mathcal{V}(I)$ of $\mathcal{Z}$ is Poisson closed if its defining ideal is Poisson.

Extension $(e: J \longrightarrow J H)$ and contraction $\left(c: I \longrightarrow I \cap Z_{0}\right)$ are mappings between the ideals of $Z_{0}$ and $H$, which map Poisson ideals to Poisson ideals. It is an easy exercise using Going Up [19, 10.2.10(ii)] to show that

$$
c \circ e \text { is the identity on semiprime ideals of } Z_{0} \text {. }
$$

Clearly, therefore, if $I$ is a semiprime Poisson ideal of $Z_{0}$ then there is an induced structure of Poisson $Z_{0} / I$-order on $A / I A$, and if $J$ is a Poisson ideal of $H$ then there is an induced structure of Poisson $Z_{0} / J \cap Z_{0}$-order on $H / J$.

Note for future use that it's trivial to check the following:

Lemma. Assume the hypotheses and notation of (5.5). If $I$ is a Poisson ideal of $H$ or of $Z_{0}$ then $\operatorname{gr} I$ is a Poisson ideal of $\operatorname{gr} H$ or of $\operatorname{gr} Z_{0}$, respectively.

5.8. Symplectic Leaves. As we shall see, the symplectic leaves of $\mathcal{Z}$ provide a key too in the analysis of a Poisson $Z_{0}$-algebra $H$. We begin in this paragraph with a Poisson $\mathbb{C}$-algebra $Z_{0}$ - that is, we are in the commutative world for now. Each $f \in Z_{0}$ defines a Hamiltonian vector field $\{f,-\}$. Suppose for a moment that $\mathcal{Z}$ is smooth, and let $\mathfrak{m}$ be a maximal ideal of $Z_{0}$. Then the symplectic leaf 
containing $\mathfrak{m}$ is the maximal connected submanifold $\mathcal{L}(\mathfrak{m})$ of $\mathcal{Z}$ such that $\mathfrak{m} \in \mathcal{L}(\mathfrak{m})$ and the Hamiltonian vector fields span the tangent space at each point of $\mathcal{L}(\mathfrak{m})$.

This definition can be extended to arbitrary (not necessarily smooth) varieties $\mathcal{Z}$; for the details, see [4]. We shall say that the leaves are algebraic if each leaf is locally closed-meaning that it is an open subset of its (Zariski) closure in $\mathcal{Z}$. A particularly tractable case occurs when there are only finitely many leaves in $\mathcal{Z}$. To state the result, define the Poisson core $\mathcal{P}(\mathfrak{m})$ of a maximal ideal $\mathfrak{m}$ of $Z_{0}$ to be the (unique) largest Poisson ideal contained in $\mathfrak{m}$. By (5.7), $\mathcal{P}(\mathfrak{m})$ is a prime ideal.

Proposition. [4] Let $Z_{0}$ be a (finitely generated, commutative) $\mathbb{C}$ algebra, and set $\mathcal{Z}=\operatorname{maxspec} Z_{0}$.

1. There are only finitely many leaves in $\mathcal{Z}$ if and only if (a) the leaves are algebraic and (b) the Poisson spectrum $\mathcal{P}-\operatorname{spec}\left(Z_{0}\right)$ of $Z_{0}$ is finite.

2. Suppose that there are only finitely many leaves in $\mathcal{Z}$. Let $\mathfrak{m} \in \mathcal{Z}$. Then

$$
\mathcal{L}(\mathfrak{m})=\{\mathfrak{n} \in \mathcal{Z}: \mathcal{P}(\mathfrak{n})=\mathcal{P}(\mathfrak{m})\},
$$

and $\mathcal{L}(\mathfrak{m})$ consists precisely of the smooth points in the closed subset of $\mathcal{Z}$ defined by $\mathcal{P}(\mathfrak{m})$.

Of particular relevance in geometric applications is the following corollary; here, $3 \Longrightarrow 2 \Longrightarrow 1$ is immediate from the definition of a leaf and the proposition, but some further argument is needed for the remaining implication. Naturally, we say that a Poisson algebra is Poisson simple if it has no proper Poisson ideals.

Corollary. Let $Z_{0}$ be a (finitely generated, commutative) $\mathbb{C}$-algebra which is a domain, and set $\mathcal{Z}=\operatorname{maxspec} Z_{0}$. Suppose that there are only finitely many leaves in $\mathcal{Z}$. Then the following are equivalent:

1. $\mathcal{Z}$ is smooth;

2. there is only one leaf in $\mathcal{Z}$;

3. $Z_{0}$ is Poisson simple.

5.9. Leaves in Symplectic Reflection Algebras-the Classical Case. Fix $(V, \omega, \Gamma)$ as in (5.4)1. We shall describe the symplectic leaves of $V / \Gamma$. In particular, we'll see that they are finite in number, so that Proposition (5.8) applies. 
Given $v \in V$, we let $\Gamma_{v}=\{\gamma \in \Gamma: \gamma v=v\}$, the stabiliser of $v$, and given $T \leq \Gamma$, we let $\mathcal{V}_{T}^{o}=\left\{v \in V: T=\Gamma_{v}\right\}$, and $\mathcal{V}_{T}=\left\{v \in V: T \subseteq \Gamma_{v}\right\}$. Let $I(T)=\left\{x^{h}-x: x \in S(V), h \in T\right\}$, an ideal of $S(V)$, and set

$$
J(T)=I(T) \cap S(V)^{\Gamma}=\bigcap_{\gamma \in \Gamma} I\left(T^{\gamma}\right) \cap S(V)^{\Gamma},
$$

an ideal of $S(V)^{\Gamma}$. Clearly $\mathcal{V}_{T}$ is a closed subset of $V$ with $I\left(\mathcal{V}_{T}\right)=$ $I(T)$, and $\mathcal{V}_{T}^{o}$ is open in $\mathcal{V}_{T}$, being the complement in $\mathcal{V}_{T}$ of the closed subset of points with stabiliser strictly containing $T$. Letting $T$ vary over subgroups of $\Gamma$ thus gives a stratification of $V$ by locally closed subsets,

$$
V=\coprod_{T \leq \Gamma} \mathcal{V}_{T}^{o}
$$

Let $\pi: V \longrightarrow V / \Gamma$ be the orbit map, and for $T \leq \Gamma$ set $\mathcal{Z}_{T}^{o}=\pi\left(\mathcal{V}_{T}^{o}\right)$, a locally closed subset of $V / \Gamma$ which depends only on the conjugacy class of $T$ in $\Gamma$. So there is a stratification of $V / \Gamma$ by the locally closed sets $\mathcal{Z}_{T}^{o}$,

$$
V / \Gamma=\coprod_{T \leq \Gamma} \mathcal{Z}_{T}^{o}
$$

and

$$
\mathcal{Z}_{T}:=\overline{\mathcal{Z}}_{T}^{o}=\pi\left(\mathcal{V}_{T}\right)
$$

with $J(T)$ being the defining ideal of $\mathcal{Z}_{T}$.

Let's do the easy calculation to see that $J(T)$ is a Poisson ideal, or equivalently that $\mathcal{Z}_{T}$ is a Poisson closed subset of $\mathcal{Z}$. Take $x, x^{\prime} \in$ $S(V), y \in S(V)^{\Gamma}$ and $h \in T$. Then, since the Poisson bracket is induced from the symplectic form on $V$,

$$
\begin{aligned}
\left\{\left(x^{h}-x\right) x^{\prime}, y\right\} & =\left\{x^{h}-x, y\right\} x^{\prime}+\left(x^{h}-x\right)\left\{x^{\prime}, y\right\} \\
& =\left(\{x, y\}^{h}-\{x, y\}\right) x^{\prime}+\left(x^{h}-x\right)\left\{x^{\prime}, y\right\},
\end{aligned}
$$

proving that $I(T)$, and therefore $J(T)$, is stable under the Poisson action of $S(V)^{\Gamma}$.

In fact, with more work one can show that the different ideals $J(T)$ are the only prime Poisson ideals of $S(V)^{\Gamma}$. In other words:

Proposition. [4] The symplectic leaves of $V / \Gamma$ are precisely the sets $\mathcal{Z}_{T}^{o}$ as $T$ runs through the conjugacy classes of subgroups of $\Gamma$ for which $\mathcal{V}_{T}^{o} \neq \emptyset$. 
Example: Consider once again the Kleinian singularity of type $A_{n-1}$ of $(2.2)$. So here $I(\{1\})=0$ and $I(\{T\})=\langle V\rangle$ for every nonidentity subgroup $T$ of $\Gamma$. Thus the proposition tells us that there is only one proper Poisson prime ideal of $S(V)^{\Gamma}$, namely $\left\langle x^{n}, y^{n}, x y\right\rangle$, and that the stratification of $V / \Gamma$ into symplectic leaves is

$$
V / \Gamma=\pi(\{0\}) \cup \pi(V \backslash\{0\}) .
$$

5.10. Leaves in Symplectic Reflection Algebras-the Quantized Case. Now suppose that we are in the deformed setting of (5.4)2. Analogously to the classical case, we have the

Proposition. [4] Let $H_{0, \mathbf{c}}$ be a symplectic reflection algebra with centre $Z_{0, \mathbf{c}}$ as in (5.4)2. Then $\operatorname{maxspec}\left(Z_{0, \mathbf{c}}\right)$ has only finitely many symplectic leaves.

Unfortunately, there is (at least at present) no precise description of the leaves in the quantised case, such as we have for the classical case. Each leaf in the quantum setting is associated, via Proposition (5.8) and the filtered-graded process of Lemma (5.5), with a semiprime Poisson ideal of $S(V)^{\Gamma}$, and this association is wellbehaved with respect to dimension. The semiprime Poisson ideals of $S(V)^{\Gamma}$ are known, thanks to Proposition (5.9), but we have no information as to whether the correspondence just described distinguishes the distinct leaves of maxspec $\left(Z_{0, \mathbf{c}}\right)$. More detailed information of this sort would be extremely valuable from the perspective of symplectic algebraic geometry, to give but one application. For, one important open question is to understand for which symplectic reflection algebras it is the case that $Z_{0, \mathbf{c}}$ is smooth for some (and so for a generic) choice of the parameters c. This is because when $Z_{0, \mathbf{c}}$ is smooth it is hoped that it will afford a symplectic resolution of singularities of $V / \Gamma$ as defined in (3.4). For definitions and a full discussion, see [11].

In the particularly significant case of the rational Cherednik algebras, namely those symplectic reflection algebras constructed from a dual pair $(U, W)$ as in (3.2)2, with $\mathrm{U}$ the Cartan subalgebra of a complex simple Lie algebra and $W$ its Weyl group, $Z_{0, \mathbf{c}}$ is smooth for a generic choice of $\mathbf{c}$ when $W$ has type $\mathrm{A}$ or $\mathrm{B}$, but $Z_{0, \mathbf{c}}$ is never smooth when $W$ has type $G_{2}[11$, Section 16]. It's very suggestive to compare this with Theorem (3.4)3. 
5.11. The Non-PI Case. Recall from Theorem (4.5) that $H_{t, \mathbf{c}}$ satisfies a polynomial identity if and only if $t=0$. When $t$ is non-zero $Z\left(H_{t, \mathbf{c}}\right)=\mathbb{C}$ by Theorem (4.5)4, and we expect that $H_{t, \mathbf{c}}$ should resemble $H_{1, \mathbf{0}}$, a simple ring. For example, in the Kleinian case (3.2)1 studied by Crawley-Boevey and Holland it is known [5] that, for $t \neq 0, H_{t, \mathbf{c}}$ has only finitely many ideals, and indeed is simple for generic values of $t$ and $\mathbf{c}$. Naturally therefore one asks:

Question: Are the above conclusions still valid when $\operatorname{dim}_{\mathbb{C}}(V)>2$ ?

This question is closely related to the Poisson structure of $H_{0, \mathbf{0}}$, thanks to the

Lemma. If $t \neq 0$ and $I$ is an ideal of $H_{t, \mathbf{c}}$ then $\operatorname{gr} I$ is a Poisson ideal of $H_{0,0}$.

Proof. The filtration on $H_{t, \mathbf{c}}$ is that defined in (4.1). Let $\alpha \in H_{0,0}$ and let $z \in Z\left(H_{0,0}\right)=S(V)^{\Gamma}$. Choose $\hat{\alpha}$ and $\hat{z}$ with $\sigma(\hat{\alpha})=\alpha$ and $\sigma(\hat{z})=z$, where $\sigma$ denotes the principal symbol map. As in (5.3), defining

$$
\{z, \alpha\}:=\sigma([\hat{z}, \hat{\alpha}])
$$

yields a structure of Poisson $S(V)^{\Gamma}$-algebra on $H_{0, \mathbf{0}}$. However, this Poisson structure can be shown to have degree -2 , and so, as in (5.6), it is a non-zero scalar multiple of the restriction of the standard bracket on $S(V)$ to $S(V)^{\Gamma}$. From (10) it's clear that if $\alpha \in \operatorname{gr} I$ then $\{z, \alpha\} \in \operatorname{gr} I$, as required.

While the above lemma does provide some information on the ideal structure of $H_{t, \mathbf{c}}$ for $t \neq 0$, it's unsatisfactory in several respects. First, one has the same problem regarding the "fibres" of the map from ideals to Poisson ideals which we encountered in (5.10); and secondly, it would be preferable to relate ideals of $H_{t, \mathbf{c}}$ to Poisson ideals of $H_{0, \mathbf{c}}$ rather than of $H_{0, \mathbf{0}}$. At present we have nothing positive to report on either of these problems.

\section{Representation Theory}

6.1. Walking Across a Leaf. The (finite dimensional) representation theory of the algebras $H_{0, \mathbf{c}}$ is intimately connected to the symplectic geometry of their centres $Z_{0, \mathbf{c}}$, as is made clear by the following result. A version of this theorem was first proved by De Concini and Lyubashenko for use in their study of quantised function 
algebras at a root of unity [6]; see also [7]. In those versions the central subalgebra $Z_{0}$ of the Poisson $Z_{0}$-order $H$ being studied had to be smooth, with $H$ a projective $Z_{0}$-module. The present version, due to Brown and Gordon [4], avoids those hypotheses, but does require that the leaves are algebraic. Its proof consists in making various reductions so as to pass to a setting where the additional hypotheses needed for the argument of De Concini and Lyubashenko hold. The key point of the latter is to integrate the Hamiltonian vector fields to get a linear isomorphism, which is then shown to be an algebra homomorphism.

Theorem. Let $H$ be an affine $\mathbb{C}$-algebra which is a Poisson $Z_{0^{-}}$ order (as defined in (5.1)). Suppose that the symplectic leaves of $\operatorname{maxspec}\left(Z_{0}\right)$ are algebraic. If $\mathfrak{m}$ and $\mathfrak{n}$ are maximal ideals of $Z_{0}$ which belong to the same leaf, then

$$
H / \mathfrak{m} H \cong H / \mathfrak{n} H .
$$

Let's consider for a moment why the above result is significant for the representation theory of $H$. If $W$ is an irreducible $H$-module then $\operatorname{End}_{H}(W)=\mathbb{C}$ by a version [19, Proposition 9.1.7] of Schur's lemma. Hence, (defining $\operatorname{Ann}_{Z_{0}}(W)=\left\{z \in Z_{0}: z W=0\right\}$ ), it follows that $Z_{0} / \operatorname{Ann}_{Z_{0}}(W)=\mathbb{C}$. That is, $\operatorname{Ann}_{Z_{0}}(W):=\mathfrak{m}$ is a maximal ideal of $Z_{0}$ and $W$ is an irreducible $H / \mathfrak{m} H$-module. Moreover, if $U$ is a second irreducible $H$-module with $\operatorname{Ann}_{Z_{0}}(U)=\mathfrak{m}^{\prime}$, and there is a non-split extension $X$ of $W$ by $U$, then it's an easy exercise to show that $\mathfrak{m}=\mathfrak{m}^{\prime}$ and either $\mathfrak{m} X=0$ or $W=U$.

In other words, the finite dimensional representation theory of $H$ reduces in large part to the study of the finite dimensional algebras $H / \mathfrak{m} H$, as $\mathfrak{m}$ ranges through $\operatorname{maxspec}\left(Z_{0}\right)$. Hence the relevance of Theorem (6.1). Note that the significance of the theorem will be particularly marked when there are only finitely many leaves in $\operatorname{maxspec}\left(Z_{0}\right)$, as is the case for symplectic reflection algebras by (5.10); or when there are only finitely many orbits of leaves under the action of the group of Poisson automorphisms of $H$, as is the case for quantised function algebras at a root of unity [6].

6.2. Azumaya Strata. Let's see what Theorem (6.1) tells us in the simplest setting. So we assume that we have a prime Poisson $Z_{0^{-}}$ order $H$ with $Z_{0}=Z(H)$; and we assume that there are only finitely many leaves in maxspec $\left(Z_{0}\right)$ (although much of what follows is true under weaker hypotheses). 
Recall that if $R$ is a prime algebra which is a finite module over its $\mathbb{C}$-affine centre $Z$, then a maximal ideal $\mathfrak{m}$ of $Z$ is called an Azumaya point of maxspec $(Z)$ if $R_{\mathfrak{m}}$ is an Azumaya $Z_{\mathfrak{m}}$-algebra, meaning that $R_{\mathfrak{m}}$ is a free $Z_{\mathfrak{m}}$-module with

$$
R_{\mathfrak{m}} \otimes_{Z_{\mathfrak{m}}} R_{\mathfrak{m}}^{\text {op }} \cong \operatorname{End}_{Z_{\mathfrak{m}}}\left(R_{\mathfrak{m}}\right),
$$

$[19,13.7 .6]$. Thanks to the Artin-Procesi theorem [19, Theorem 13.7.14] this is equivalent to requiring that

$$
R / \mathfrak{m} R \cong M_{n}(\mathbb{C}),
$$

(where $n$ is the PI-degree of $R$ ). In other words, the Azumaya points of $\operatorname{maxspec}(Z)$ are precisely the points $\mathfrak{m}$ at which the structure of $R / \mathfrak{m} R$ is as simple as possible; such points are generic in maxspec(Z), [19, Theorem 13.7.14(iii)]. The Azumaya locus $\mathcal{A}_{Z}$ of maxspec $(Z)$ is the open dense subset of Azumaya points of $\operatorname{maxspec}(Z)$, and we call $R$ an Azumaya algebra if $\mathcal{A}_{Z}=\operatorname{maxspec}(Z)$. Now we're ready to state a noncommutative addition to the equivalences of Corollary (5.8). The first part is immediate from that corollary and Theorem (6.1).

Corollary. Let $H$ be a prime Poisson $Z$-order, where $Z$ is the centre of $H$. Assume that there are only finitely many leaves in $\mathcal{Z}=\operatorname{maxspec}(Z)$.

(a) The following are equivalent:

1. $\mathcal{Z}$ is smooth;

2. there is only one leaf in $\mathcal{Z}$;

3. $Z$ is Poisson simple;

4. $\mathrm{H}$ is an Azumaya algebra.

(b) Assume that $H$ has finite global (homological) dimension. Then (generalising (a)), the locus of smooth points of $\mathcal{Z}$ is equal to the Azumaya locus $\mathcal{A}_{Z}$.

Proof. (b) By the last part of Proposition (5.8), the smooth points of $\mathcal{Z}$ belong to a single leaf. Since this set is dense in $\mathcal{Z}$ it must meet the dense set $\mathcal{A}_{Z}$. By Theorem (6.1) we deduce that every smooth point of $\mathcal{Z}$ is Azumaya. Conversely, let $\mathfrak{m}$ be an Azumaya point of $\mathcal{Z}$. Then $H_{\mathfrak{m}}$ is a projective $Z_{\mathfrak{m}}$-module, so, by the hypothesis on the global dimension of $H$ it follows that $Z_{\mathfrak{m}}$ has finite global dimension. That is, $\mathfrak{m}$ is a smooth point, as required. 
Comments: 1. I've chosen to highlight part (a) of the corollary because - when applied to the symplectic reflection algebras $H_{0, \mathbf{c}}$ it makes precise the way in which their noncommutative structure encodes the geometry of the varieties whose coordinate rings are the algebras $Z_{0, \mathbf{c}}$; and recall that the latter are "symplectic deformations" of $V / \Gamma$.

2. The same conclusion as in part (b) of the corollary is obtained in an earlier theorem of Lebruyn [16, Proposition 5], Brown and Goodearl [3, Theorem 3.8]. In the earlier result no Poisson structure is involved, but much heavier homological conditions have to be imposed on $H$, and one has to assume that the complement of $\mathcal{A}_{Z}$ has codimension at least 2 in $\mathcal{Z}$. (In Corollary (6.2)(b) the latter condition is (implicitly) a consequence of the Poisson structure.)

3. The corollary is the "first step" in a stratification of $\mathcal{Z}$ one factors $H$ by the ideal generated by the ideal of $Z$ defining the complement of the Azumaya locus $\mathcal{A}_{Z}$ - this is a Poisson ideal - and repeats the game in the factor algebra. In this way one can realise $H$ as a sheaf of Azumaya algebras - for details, see [4]. This sort of approach was pioneered in the case of quantised function algebras at a root of 1 by De Concini and Procesi [8].

\section{REFERENCES}

[1] D.J.Benson, Polynomial Invariants of Finite Groups, London Math. Soc. Lecture Note Series, 190, CUP, 1993.

[2] K.A. Brown and K.R.Goodearl, Lectures on Algebraic Quantum Groups, Birkhauser, 2002.

[3] K.A. Brown and K.R.Goodearl, Homological aspects of noetherian PI Hopf algebras and irreducible modules of maximal dimension, J. Algebra 198(1) (1997), 240-265.

[4] K.A. Brown and I. Gordon, Poisson orders, symplectic reflection algebras and representation theory, arXiv math.RT/0201042, to appear in J. fur die Reine und Angewandte Mathematik.

[5] W. Crawley-Boevey and M. Holland, Noncommutative deformations of Kleinian singularities, Duke Math. J. 92 (1998), 605-635.

[6] C. De Concini and Lyubashenko, Quantum function algebras at roots of 1 , Adv. Math., 108 (1994), 205-262.

[7] C. De Concini and C. Procesi, Quantum groups, pp 31-140 in D-modules, Representation Theory, and Quantum Groups, (G. Zampieri and A. D'Agnolo, eds.), Springer Lecture Notes in Mathematics 1565, Berlin, 1993.

[8] C. De Concini and C. Procesi, Quantum Schubert cells and representations at roots of 1, pp 127-160 of Algebraic groups and Lie groups (ed. G. Lehrer), Australian Math. Soc. Lecture Series 9, CUP, Cambridge, 1997. 
[9] J. Dixmier, Enveloping Algebras, Graduate Studies in Math. 11, Amer. Math. Soc. Providence, RI, 1996.

[10] P. Du Val, On isolated singularities which do not affect the conditions of adjunction, Part 1, Math. Proc. Cam. Phil. Soc. 30 (1934), 453-456.

[11] P. Etingof and V. Ginzburg, Symplectic reflection algebras, CalegoroMoser space, and deformed Harish-Chandra homomorphism, Inv. Math. 147 (2002), 243-348; arXiv:math.AG/0011114, April 2001.

[12] K.R. Goodearl and R.B. Warfield Jr., An Introduction to Noncommutative Noetherian Rings, London Mathematical Society Student Texts, 16, CUP, Cambridge, 1989.

[13] R.Guralnick and J. Saxl, Generation of finite almost simple groups by conjugates, submitted.

[14] T. Hayashi, Sugawara operators and the Kac-Kazhdan conjecture, Inv. Math. 94 (1988), 13-52.

[15] D. Kaledin, Symplectic resolutions: deformations and birational maps, arXiv:math.AG/0012008.

[16] L. Lebruyn, Central singularities of quantum spaces, J. Algebra 177 (1995), $142-153$.

[17] T. Levasseur, Some properties of noncommutative regular graded rings, Glasgow Math. J. 34 (1992), 277-300.

[18] R. Martin, Skew group rings and maximal orders, Glasgow Math. J. 37 (1995), 249-263.

[19] J.C. McConnell and J.C. Robson, Noncommutative Noetherian Rings, Wiley-Interscience, New York, 1987.

[20] D.S. Passman, Infinite Crossed Products, Pure and Applied Math vol. 135, Academic Press, San Diego, 1989.

[21] M. Van den Bergh and F. Van Oystaeyen, Lifting maximal orders, Comm. in Algebra 17 (1989), 341-349.

[22] M. Verbitsky, Holomorphic symplectic geometry and orbifold singularities, Asian J. Math. 4 (2000), no. 3, 553-563; arXiv:math.AG/9903175.

Kenneth A. Brown,

Mathematics Department,

University of Glasgow,

Glasgow G12 8QW, Scotland

kab@maths.gla.ac.uk

Received on 4 December 2001 and in revised form on 26 September 2002. 